\section{Early aging and age-related pathologies in mice deficient in BMAL1, the core component of the circadian clock}

\author{
Roman V. Kondratov, ${ }^{1,4}$ Anna A. Kondratova, ${ }^{2}$ \\ Victoria Y. Gorbacheva, ${ }^{1}$ Olena V. Vykhovanets, ${ }^{1}$ \\ and Marina P. Antoch ${ }^{1,3}$ \\ ${ }^{1}$ Department of Cancer Biology, Lerner Research Institute, \\ Cleveland Clinic Foundation, Cleveland, Ohio 44195, USA; \\ ${ }^{2}$ Department of Molecular Genetics, Lerner Research \\ Institute, Cleveland Clinic Foundation, \\ Cleveland, Ohio 44195, USA
}

Mice deficient in the circadian transcription factor BMAL1 (brain and muscle ARNT-like protein) have impaired circadian behavior and demonstrate loss of rhythmicity in the expression of target genes. Here we report that $\mathrm{Bmal1}^{-/-}$mice have reduced lifespans and display various symptoms of premature aging including sarcopenia, cataracts, less subcutaneous fat, organ shrinkage, and others. The early aging phenotype correlates with increased levels of reactive oxygen species in some tissues of the $\mathrm{Bmal1}^{-/}$animals. These findings, together with data on CLOCK/BMAL1-dependent control of stress responses, may provide a mechanistic explanation for the early onset of age-related pathologies in the absence of BMAL1.

Supplemental material is available at http://www.genesdev.org.

Received March 22, 2006; revised version accepted May 9, 2006.

BMAL1 (brain and muscle ARNT-like protein, also known as MOP3 or ARNT3) belongs to the family of the basic helix-loop-helix (bHLH)-PAS domain-containing transcription factors. In a complex with another member of this family, CLOCK, BMAL1 regulates expression of genes through E-box elements in their promoters. BMAL1, CLOCK, and some of their transcriptional targets (PERIODs and CRYPTOCHROMEs) are the key components of the molecular oscillator that generates circadian rhythms. These 24-h oscillations in behavior, physiology, and metabolism are thought to ensure adaptation of organisms to the 24-h periodicity of the Earth's rotation (Panda et al. 2002b; Lowrey and Takahashi 2004). Although the master circadian clock is located within the hypothalamic suprachiasmatic nucleus (SCN), the key circadian proteins are expressed in many peripheral tissues, determining circadian periodicity in

[Keywords: BMAL1; circadian clock; aging]

Corresponding authors.

${ }^{3}$ E-MAIL antochm@ccf.org; FAX (216) 445-6269.

${ }^{4}$ E-MAIL kondrar@ccf.org; FAX (216) 445-6269.

Article is online at http://www.genesdev.org/cgi/doi/10.1101/gad.1432206. gene expression and physiology for many organs (Reppert and Weaver 2002). Indeed, microarray data show that up to $10 \%$ of genes in different tissues are directly or indirectly regulated by the circadian clock system (Panda et al. 2002a; Storch et al. 2002). At the same time, only a few oscillating genes (most of them encoding components of the molecular clock itself) are common to all organs tested. Since different tissues have characteristic sets of genes with clock-regulated timing and amplitude of expression (Panda et al. 2002a; Storch et al. 2002), it is likely that the circadian clock is involved in control of homeostasis in different organs.

Mice with mutations or targeted disruptions of core circadian genes that have been generated during the last decade all show impaired circadian behavior and deregulation of circadian patterns in gene expression (Lowrey and Takahashi 2004). In addition to this universal phenotype, other pathological defects are specific for particular circadian mutants. Thus, Clock mutation results in reduced fertility and complications of pregnancy (Miller et al. 2004), obesity and metabolic syndrome (Turek et al. 2005), and sensitization to cocaine (McClung et al. 2005). Period2 deficiency leads to the enhanced voluntary alcohol consumption and alterations in the glutamatergic system (Spanagel et al. 2005), higher sensitivity to radiation and increased tumor formation after irradiation (Fu et al. 2002), and alterations in bone remodeling and bone mass accumulation (Fu et al. 2005). Hence, some circadian proteins play important roles in organ physiology that are not necessarily linked to their circadian function. This is certainly true for BMAL1, since $\mathrm{Bmal1}^{-/-}$mice, in addition to loss of circadian rhythms, display a number of phenotypes including infertility (Kennaway 2005), defective glucose homeostasis (Rudic et al. 2004), idiopathic calcification and ossification of hind limb joints (Bunger et al. 2005), and increased sensitivity to chemotherapy and radiation (Gorbacheva et al. 2005). To further investigate the role of BMAL1 in normal physiology, we monitored a large group of $B \mathrm{mal1}^{-/-}$knockout (KO) and wild-type mice for their entire lifespan. This revealed that Bmal1 KO animals had reduced lifespan and developed a number of pathologies characteristic of mice with premature aging phenotypes (Tyner et al. 2002; Mounkes et al. 2003; Chang et al. 2004; Trifunovic et al. 2004; Kujoth et al. 2005; Kurosu et al. 2005).

\section{Results and Discussion}

Since the genetic background markedly influences phenotype of a given single-gene mutation in mice, to address the role of BMAL1 in normal physiology, we first transferred the mutation to C56BL/6J mouse strain (12 backcross generations). Due to infertility of the mutants, $B \mathrm{mal1}^{-/-}$colony was maintained through the breeding of heterozygous animals, which produced the progeny of all three genotypes with expected ratio. KO animals and their wild-type littermates were monitored through their lifespan for the development of all kinds of pathological changes.

The lifespan of $\mathrm{KO}$ animals was significantly reduced compared with their wild-type littermates (Fig. 1A). Most mutants died between 26 and 52 wk of age (average 
A
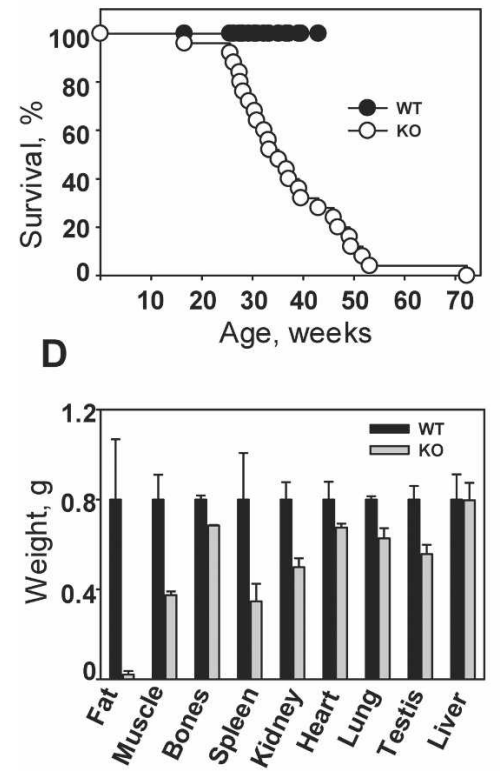

B

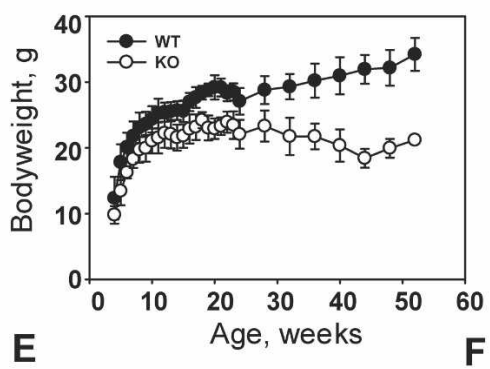

C

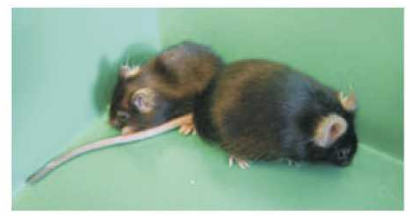

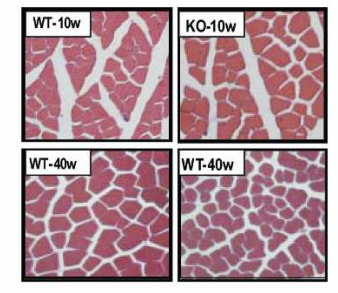

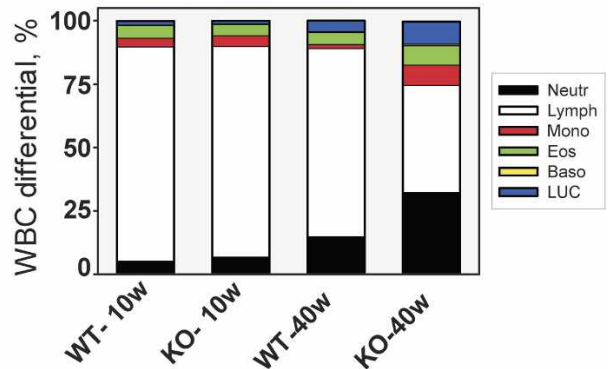

Figure 1. Premature aging and early death in Bmal1 KO mice. (A) Kaplan-Meyer survival curve. (Closed circles) Wild-type (WT) mice; (open circles) KO mice. Wild-type animals older than 1 yr were sacrificed. (B) Age-dependent changes in body weight of wild-type (WT) and KO males. (Closed circles) Wild-type mice; (open circles) KO mice. (C) Gross appearance of 1-yr-old wild-type (WT) (right) and KO (left) male mice. (D) Weight of major organs from the 40-wk-old mice. (Black bars) Wild type (WT); (gray bars) KO. All organs from KO animals, with the exception of liver, are significantly smaller than wild type $(p<0.05)$. $(E)$ The diameter of muscle fibers is reduced in $B$ mal1 ${ }^{-1-}$ animals. Hematoxylin and eosin (H\&E) staining of cross-sections of femoral quadriceps from 10- and 40-wk-old animals of both genotypes. $(F)$ WBC differentials in young (10-wk-old) and aged (40-wk-old) wild-type (WT) and KO animals. Each stack in the bar represents cell type percentage. (Neutr) Neutrophils; (Lymph) lymphocytes; (Mono) monocytes; (Eos) eosinophils; (Baso) basophils; (LUC) large unstained cells. In aged KO mice, the percentage of lymphocytes is decreased and the percentage of monocytes/neutrophils is increased.

lifespan $37.0 \pm 12.1 \mathrm{wk}$ ), while none of the wild-type or heterozygous mice died during the period of the study (52 wk). The average lifespan of C57BL/6J animals exceeds 2 yr (Nadon 2006); more than twofold longer than that observed for the KO animals. The longest living mutant died at $72 \mathrm{wk}$ of age, which is approximately twofold shorter than the maximum lifespan in this strain. No sex-dependent differences in mortality were observed.

At birth, the KO animals do not display any visible defects and are indistinguishable from their wild-type littermates. However, at 16-18 wk of age (for males and females, respectively) $\mathrm{Bmal1}^{-/-}$mice begin to show growth retardation, as evidenced by reduced body weight. While the weight of wild-type animals increased constantly during the $52 \mathrm{wk}$ of the study, the weight of mutants stopped increasing at 16-26 wk of age, and then started to decline (Fig. 1B; Supplementary Fig. 1). As a result, 52-wk-old KO mice are about half the size of wildtype mice (Fig. 1C).

To investigate the cause of the body weight loss observed in KO animals, we measured the mass of major organs and tissues from 10-, 30-, and 40-wk-old animals of both genotypes. At 10-wk of age, all major organs from wild-type and $\mathrm{KO}$ mice were equivalent in size, with the exception of the seminal vesicles, which were smaller in Bmal1-/- animals. This reduction, which becomes more pronounced with age, likely reflects defective sexual maturation in $\mathrm{KO}$ mice, leading to male infertility.

The differences in total body weight of 40-wk-old wild-type and $\mathrm{KO}$ animals were found to be primarily due to decreased adipose and muscle tissue mass in the KO mice (Fig. 1D). The average mass of intra-abdominal adipose tissue was $1.5 \mathrm{~g}$ in 40-wk-old wild-type males, but only $70 \mathrm{mg}$ in age- and sex-matched KOs. The subcutaneous fat layer was also significantly reduced in mutants (Fig. 2). This phenotype is in striking contrast to the predisposition to obesity observed in Clock/Clock mutant mice (Turek et al. 2005). There are several possible explanations for these contrasting phenotypes. The CLOCK/BMAL1 transcriptional complex cannot be formed in Bmal1-deficient cells, while in Clock/Clock cells, mutant CLOCK protein retains the ability to dimerize with BMAL1. As it has been reported recently, the CLOCK/BMAL1 complex displays dual functional activity, working both as transcriptional activator and transcriptional repressor, depending on its interaction with CRY. As a consequence, both functions of the complex are lost in Bmal1-deficient cells, while in Clock mutant cells only transactivation is impaired (Kondratov et al. 2006b). Alternatively, the observed phenotypic differences between Bmal1-- and Clock/Clock mice might be explained by CLOCK and BMAL1 interaction with different functional partners.

Forty-week-old $\mathrm{Bmal1}^{-/-}$animals also demonstrate age-dependent reduction of muscle and bone mass (Fig. 1D). Both phenomena (sarcopenia of aging and osteoporosis) are hallmarks of mammalian aging (Weiss et al. 1991; Karakelides and Sreekumaran Nair 2005). Histological examination of cross-sections of the femoral quadriceps muscle showed that the reduced muscle mass in $\mathrm{KO}$ animals is associated with a decrease in the num- 
A

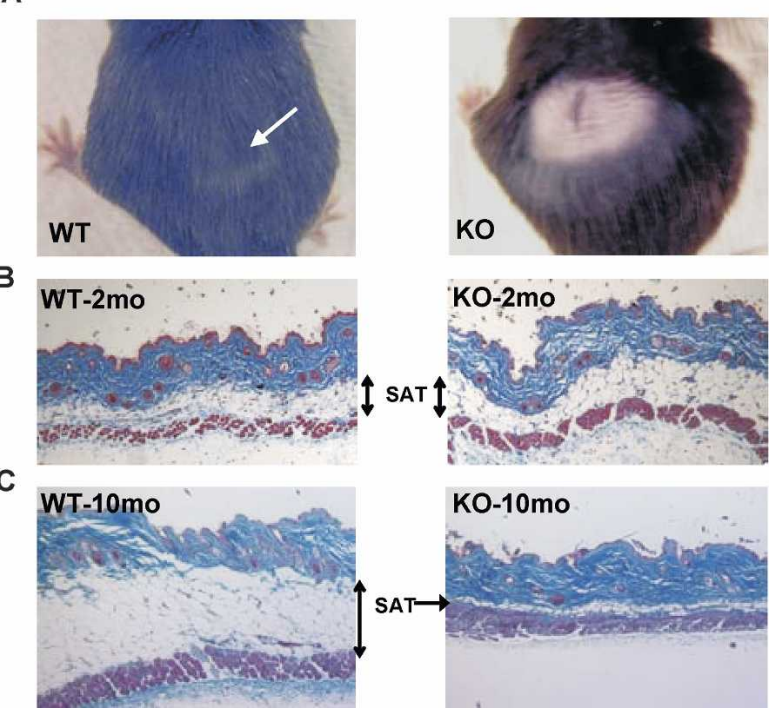

Figure 2. Age-related pathologies of the skin in KO mice. $(A)$ Dorsal hair regrowth $1 \mathrm{mo}$ after shaving was observed in wild-type (WT) (left) but not KO (right) mice. (B) H\&E staining of skin cross-sections from 10-wk-old wild-type (WT) and KO mice does not reveal any morphological differences between genotypes. (SAT) Subcutaneous adipose tissue. $(C) \mathrm{H} \& \mathrm{E}$ staining of skin cross-sections shows a dramatic reduction in SAT in 40-wk-old KO mice.

ber of muscle fibers and their diameter. This defect is age dependent, as 10-wk-old animals do not show any differences in the structure of muscle fibers between genotypes (Fig. 1E).

In addition to fat, muscle, and bone, the mass of several major organs (spleen, kidney, testis, heart, and lung) was significantly reduced in 40 -wk-old $\mathrm{KO}$ animals (Fig. 1D). At the same time, there were no differences in stomach, liver, or intestine size between the genotypes. At 30-wk of age, only the spleen was significantly smaller in $\mathrm{KO}$ as compared with wild-type animals (Supplementary Fig. 2). All other organs were just at the lower border of their normal weight range. Histological examination of $\mathrm{KO}$ spleen and kidney did not reveal any degenerative lesions (data not shown), suggesting that their smaller size is not due to an acute degenerative process, but is rather a consequence of systematic reduction. Age-associated shrinkage of spleen, kidney, and testis has been observed during natural aging as well as in animal models of accelerated aging (Arking 1998; Tyner et al. 2002).

Bmal1 ${ }^{-1-}$ mice also demonstrate age-related changes in peripheral blood composition. Total blood cell counts in young (10-wk-old) and aged/adult (40-wk-old) KO and wild-type mice show no differences in the number of red blood cell or platelets. The number of white blood cells (WBC) was identical in young animals of both genotypes and declined with age in a similar way as expected (Razzaque et al. 2006); however, this decline was slightly more pronounced in the KO mice (Supplementary Fig. 3). More prominent BMAL1-dependent changes were observed in the WBC differentials. The total number and percentage of neutrophils and monocytes in 40-wk-old Bmal1 ${ }^{-1-}$ mice were almost twofold greater than in agematched controls, while the number and percentage of lymphocytes were decreased (Fig. 1F).
Another hallmark of mammalian aging, decreased hair growth, was also observed in Bmal1 ${ }^{-/-}$mice (Harrison and Archer 1988; Tyner et al. 2002). When dorsal segments of skin were shaved on 30-wk-old animals, all four wild-type mice tested showed complete regrowth in one month. In contrast, only one of five KOs demonstrated partial regrowth after 3 mo (Fig. 2A). Similar to the other phenotypes described, this is clearly an age-related defect, since 10 -wk-old KO mice displayed robust hair regrowth after shaving (data not shown).

A reduction in subcutaneous adipose tissue (SAT) is the major marker of aged skin in many mammals, including mice and humans (Chuttani and Gilchrest 1995). Histological examination of cross-sections of dorsal skin from 10-wk-old wild-type and KO mice showed no significant differences in skin structure (Fig. 2B). In contrast, the skin of the 40-wk-old KO mice displayed a striking reduction in SAT (Fig. 2C). While this phenotype might be explained by a role for BMAL1 in adipocyte differentiation in cell culture as was recently reported (Shimba et al. 2005), it is more likely that it reflects an age-dependent process, since there is no difference in SAT in young animals. In addition, normal amounts of adipose tissue were present at other sites, including eyehole, footpad, and bone marrow in $\mathrm{KO}$ animals of all ages (data not shown).

Additional pathological changes were detected in the eyes of Bmal1 ${ }^{-/-}$animals as early as 18 -wk of age. By 30 -wk of age, all $\mathrm{KO}$ mice developed various grades of cataracts in one or both eyes (Supplementary Fig. 4), while none of the age-matched wild-type littermates displayed any visible eye pathology (Fig. 3A). Histological examination of the lens posterior zone of KO mice (Fig. 3B) revealed aberrantly enlarged infiltrating epithelial cells, which are normally located only in the anterior zone of the lens (Supplementary Fig. 5). In addition, the more eosinophilic lenses of the mutants (Fig. 3A) reflect

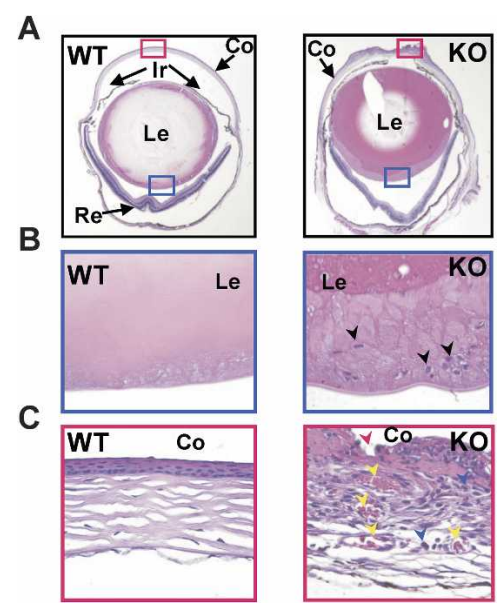

Figure 3. Age-related eye pathologies in $\mathrm{Bmal1}^{-/-}$mice. H\&E staining of cross-sections of eyes from 30-wk-old wild-type (WT) and KO mice. (Co) Cornea; (Ir) iris; (Le) lens; (Re) retina. (A) Pathological changes of the cornea and lens of KO mice. (B) Magnification of the lens posterior zone (blue rectangle in $A$ ). The arrowheads point to the nuclei of epithelial cells infiltrating the posterior zone of the KO lens. $(C)$ Magnification of the indicated zone of cornea (red rectangle in A). Acute cornea inflammation in the KO mice is characterized by neovascularization (yellow arrowheads), lymphoid cell infiltration (blue arrowheads), and ulcer (red arrowhead). 
a higher density of cortical and nucleus fibers, which is typical of aged lenses. Progressive cornea inflammation was observed in $>50 \%$ of 40 -wk-old Bmal1-deficient animals. This inflammation is initially manifested by massive neovascularization, lymphoid cell infiltration, and ulcer of the outer layer of the cornea (Fig. 3C). It progresses later to chronic cornea inflammation, in which disruption of stromal structure is observed (Supplementary Fig. 6A) together with hyperplasia of corneal epithelium and keratin deposition (Supplementary Fig. 6B). While cornea inflammation may lead to lesions in the anterior zone of lenses and initiate cataract development, we did not detect any correlation between these two pathologies.

Taken together, the spectrum of phenotypes observed in $\mathrm{Bmal1}^{-1-}$ mice as they age suggests that BMAL1 plays an important role in maintaining tissue homeostasis. In addition to disruption of circadian function, Bmal1 deficiency results in a progeria-like phenotype, which directly links this transcription factor to control of the aging process.

Dozens of theories have been put forth implying deregulation of various pathways as the major cause of aging. Despite their differences, most current theories consider oxidative stress a crucial factor associated with degenerative processes occurring during aging (Balaban et al. 2005). Reactive oxygen and nitrogen species (ROS and RNS, respectively) are important regulators of cellular metabolism, gene expression, and other molecular responses, playing key roles in the control of various physiological processes. At the same time, high concentrations of free radicals and radical-derived reactive species such as ROS and RNS have damaging effects on all major biological macromolecules. Oxidative damage may accelerate or even cause aging by contributing to age-related degenerative diseases. Indeed, it has been demonstrated that aging is associated with increased levels of ROS and oxidized products in various tissues (Balaban et al. 2005).

Having shown that BMAL1 is directly involved in modulation of stress response (Gorbacheva et al. 2005), we decided to investigate the possible involvement of BMAL1 in ROS homeostasis. To account for possible circadian variations in ROS concentration, we first measured it in several tissues of wild-type mice and have demonstrated that ROS levels display temporal variations in the liver, to a lesser extent in the kidney, and do not change in the spleen (Supplementary Fig. 7). Based on this, all of the comparisons between wild type and KO were done using tissue samples collected at the time of the highest daily levels of ROS in the liver and kidney of wild-type animals (ZT10). As shown in Figure 4A, ROS accumulation in 40-wk-old Bmall-deficient animals is significantly higher than wild type in kidney, heart, and spleen, while it is lower than wild type in liver. Importantly, ROS accumulation correlated with the observation of age-dependent pathologies in specific tissues: Kidney, heart, and spleen all demonstrate age-dependent reduction in size in $\mathrm{KO}$ animals, whereas liver does not show any age-related pathology (Fig. 1). The age-dependent nature of ROS accumulation was confirmed by comparing ROS levels in kidney and spleen of 10-, 30-, and 40-wk-old wild-type and KO mice (Fig. 4B,C). ROS accumulation in these $\mathrm{KO}$ tissues progresses with age and thus correlates with their observed age-related degeneration.

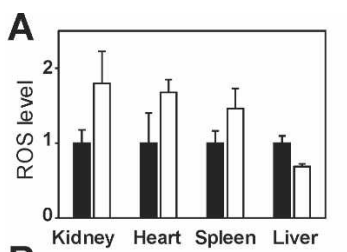

B
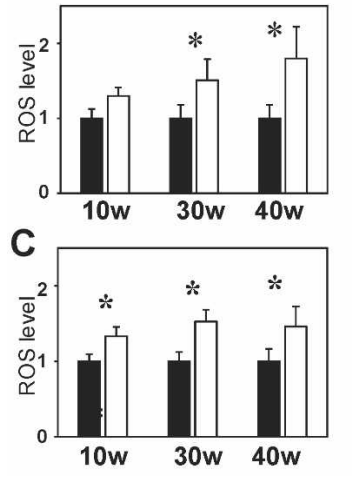

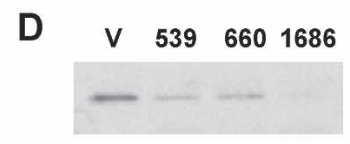

E

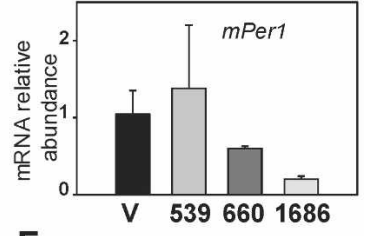

F

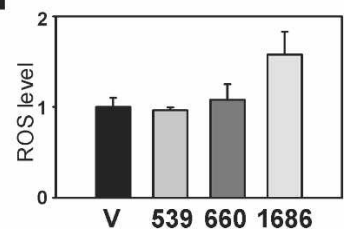

Figure 4. BMAL1 is involved in regulation of ROS levels. $(A)$ ROS levels indicated by relative DCF fluorescence in tissue extracts from 30-wk-old wild-type (WT) and KO mice. $(B, C)$ Age-dependent increases in the level of ROS in kidney $(B)$ and spleen $(C)$ of $\mathrm{KO}$ mice. At least four animals of each age group and genotype were used. ${ }^{\star}$ ) $p<0.05$. (D) BMAL1 suppression by siRNA. Western blot of lysates from L929 cells infected with a control lentivirus (V) or three different siBmal1 lentiviral constructs $(539,660$, and 1686) probed with a BMAL1-specific antibody. $(E, F)$ BMAL1 suppression by siBmal1686 results in down-regulation of Per1 gene expression $(E)$ and upregulation of ROS levels $(F)$.

To demonstrate direct involvement of BMAL1 in ROS homeostasis, we have generated three stably transfected L929 cell lines, in which endogenous BMAL1 expression is suppressed by specific small interfering RNAs (siRNAs), and compared the ROS levels in these lines with the parental one. Western blot analysis showed that BMAL1 expression is reduced in all three stable cell lines as compared with parental L929 cells, with line 1686 showing the greatest reduction (Fig. 4D). As expected, reduced levels of BMAL1 resulted in decreased expression of known BMAL1 transcriptional targets such as Period1, which showed fivefold lower expression in 1686 cells than in parental L929 cells (Fig. 4E). Importantly, the siRNA-mediated inhibition of BMAL1 caused upregulation of ROS levels (Fig. 4F). Together with previously published data implicating the circadian system in redox control (Hardeland et al. 2003), our results suggest that the circadian system is likely an important ROScontrolling system working under both normal physiological conditions and oxidative stress. Indeed, the levels of external (food-generated) and internal (metabolism/activity-generated) oxidants change during the day as a result of fluctuations in food intake and behavior. Therefore, control of ROS/RNS homeostasis by the circadian system, which is intrinsically connected to an organism's daily activity, would provide the most effective protection from the damaging effects of oxidants at any given time of day. At the same time, an imbalance between the circadian and ROS/RNS generating/metabolizing systems might increase damage due to oxidative stress, thus contributing to and/or complicating pathogenesis and aging. 
Increasing evidence has implicated the circadian system in the regulation of such processes as glucose homeostasis, obesity, bone formation, stress response, and others (Fu et al. 2002, 2005; Miller et al. 2004; Bunger et al. 2005; Gorbacheva et al. 2005; Spanagel et al. 2005; Turek et al. 2005). These findings dictate reconsideration of the role of core clock proteins and the circadian system in prevention, development, and/or progression of various pathologies. The data presented here raise an intriguing question about the interrelationship between the circadian system and aging. It is well known that the robustness of circadian rhythms decreased with age (Monk 2005). Our results demonstrate active involvement of the circadian system (or at least one of its core components, BMAL1) in the development/progression of age-related diseases. Importantly, in addition to deregulation of the circadian system in general, there is a possibility that the observed phenomenon is specific to BMAL1 deficiency. Unlike the deregulation of circadian rhythms that occurs in all circadian system mutants, the premature aging phenotype may be specific to BMAL1 deficiency. For example, Clock/Clock mutant mice and Cryptochrome double-knockout animals do not display any of the signs of premature aging found in $\mathrm{Bmal1}^{-}-$ mice (R. Kondratov, unpubl.). Nevertheless, as described above, these different circadian mutants do display unique sets of pathological changes (Garcia et al. 2000; Fu et al. 2002; Miller et al. 2004; Gorbacheva et al. 2005; McClung et al. 2005; Spanagel et al. 2005; Turek et al. 2005). Clarification of the importance of the circadian system and its various components in the aging process will be gained from detailed studies of aging in other circadian mutant mice.

The essential question raised by our data is how the Bmal1 deficiency results in development of age-related pathologies. BMAL1 control of ROS homeostasis as reported here is likely only one of the responsible pathways. Other potential mechanisms may involve BMAL1dependent control of stress response or imbalances in endocrine and/or metabolic systems that are likely under circadian control. These mechanisms, in turn, may affect ROS levels. In addition, the recently reported role of BMAL1 in glucose homeostasis and impaired glucose tolerance and insulin sensitivity in $\mathrm{Bmal1}^{-{ }^{-}}$animals (Rudic et al. 2004) suggests a possible connection between the circadian system and the insulin/insulin growth factor (IGF) pathways, which are known to be involved in the control of lifespan in different organisms (Bordone and Guarente 2005; Katic and Kahn 2005).

Aging is a complex process that is influenced by both genetic and environmental factors. Premature aging phenotypes have been described in mice with targeted disruption of genes involved in control of mutation rate, regulation of chromosome and telomere stability, ROS homeostasis, apoptosis, stress responses, and the insulin/IGF pathway (Tyner et al. 2002; Mounkes et al. 2003; Chang et al. 2004; Trifunovic et al. 2004; Kujoth et al. 2005; Kurosu et al. 2005). The mouse model of BMAL1 deficiency extends this list in a particularly interesting direction. BMAL1 is a key component of the circadian system, which is involved in the interaction of the organism with its environment; hence, it provides a unique example in which genetic disruption of the normal organism's interaction with the environment accelerates aging. The effective response of an organism to external and internal stimuli (including genotoxic stress) depends on the synchronized activity/expression of multiple genes. As a transcription factor, BMAL1 can orchestrate many processes by controling the rate-limiting components of various pathways. At the same time, BMAL1 has the potential to act through transcriptionindependent mechanisms. As we have reported recently, BMAL1 is an important regulator of post-translational modification and degradation of CLOCK, NPAS2, and presumably other proteins (Kondratov et al. 2003, 2006a).

In summary, we report here multiple phenotypic changes and pathologies in $\mathrm{Bmal1}^{-/-}$mice that are characteristic of premature aging. Future studies will clarify the role of BMAL1 and other circadian system components in the natural aging in mice and humans. Improved understanding of the interconnection between aging and the circadian clock system, as well as identification of BMAL1 transcriptional targets specifically involved in aging may ultimately lead to the development of strategies to prevent and/or treat age-related pathologies.

\section{Materials and methods}

Animals

Bmal1 ${ }^{-/}$mice were obtained from C. Bradfield (University of Wisconsin) and backcrossed to the C57BL/6J strain for 12 generations. Heterozygotes were then intercrossed to obtain animals of all three genotypes. Mice were genotyped by PCR as previously described (Bunger et al. 2000). In all experiments, wild-type littermates were used as controls. All animals were maintained on a $12 \mathrm{~h}: 12 \mathrm{~h}$ light:dark cycle. To monitor body weight gain/loss, animals were weighed once a week. Mice were observed daily to score mortality. To characterize hair growth, the backs of mice were shaved and animals were monitored for hair regrowth for $3 \mathrm{mo}$. All animal studies were conducted in accordance with the regulations of the Committee of Animal Care and Use at the Cleveland Clinic Foundation.

Total blood-cell analysis

Total blood cell analysis was performed on samples obtained through retro-orbital bleeding as previously described (Gorbacheva et al. 2005).

Tissue collection and histology

Tissue collection and histological analysis were performed using standard techniques (see Supplemental Material).

Cell culture, Western blotting, and siRNA constructs

L929 cells were maintained in Dulbecco's modified Eagle medium (DMEM) supplemented with $10 \%$ fetal bovine serum. For stable expression of siRNAs targeting Bmal1, we used recombinant lentivirus that contains a siRNA-expression cassette within the U3 region of the right LTR. The details on lentivirus generation are included in the Supplemental Material.

Measurement of ROS levels

ROS levels were determined in tissue extracts using ROS-sensitive fluorescent dye dichlorodihydrofluorescein (DCF) as previously described (Radak et al. 2004). The details of the assay are included in the Supplemental Material.

\section{Acknowledgments}

We thank Dr. P. Chumakov for providing lentiviral vectors and general expertise in the siRNA-experiments, Dr. Choogon Lee for providing BMAL1-specific antibody, and Dr. P. Stankope-Baker for editorial assistance. Acquisition of the Advia 120 instrument was made possible by a gift from Herbert and Judith Harvey. This work was supported by NIH grant CA102522 (to M.P.A.)

\section{References}

Arking, R. 1998. Biology of aging, 2nd ed. Sinauer Associates, Sunderland, MA.

Balaban, R.S., Nemoto, S., and Finkel, T. 2005. Mitochondria, oxidants, 
and aging. Cell 120: 483-495.

Bordone, L. and Guarente, L. 2005. Calorie restriction, SIRT1 and metabolism: Understanding longevity. Nat. Rev. Mol. Cell Biol. 6: 298305.

Bunger, M.K., Wilsbacher, L.D., Moran, S.M., Clendenin, C., Radcliffe, L.A., Hogenesch, J.B., Simon, M.C., Takahashi, J.S., and Bradfield, C.A. 2000. Mop3 is an essential component of the master circadian pacemaker in mammals. Cell 103: 1009-1017.

Bunger, M.K., Walisser, J.A., Sullivan, R., Manley, P.A., Moran, S.M., Kalscheur, V.L., Colman, R.J., and Bradfield, C.A. 2005. Progressive arthropathy in mice with a targeted disruption of the Mop3/Bmal-1 locus. Genesis 41: 122-132.

Chang, S., Multani, A.S., Cabrera, N.G., Naylor, M.L., Laud, P., Lombard, D., Pathak, S., Guarente, L., and DePinho, R.A. 2004. Essential role of limiting telomeres in the pathogenesis of Werner syndrome. Nat. Genet. 36: 877-882.

Chuttani, A. and Gilchrest, B.A. 1995. Skin. In Handbook of physiology, section 11: Aging (ed. J.E. Masoro), pp. 309-324. Oxford University Press, New York.

Fu, L., Pelicano, H., Liu, J., Huang, P., and Lee, C. 2002. The circadian gene Period2 plays an important role in tumor suppression and DNA damage response in vivo. Cell 111: 41-50.

Fu, L., Patel, M.S., Bradley, A., Wagner, E.F., and Karsenty, G. 2005. The molecular clock mediates leptin-regulated bone formation. Cell 122: 803-815.

Garcia, J.A., Zhang, D., Estill, S.J., Michnoff, C., Rutter, J., Reick, M., Scott, K., Diaz-Arrastia, R., and McKnight, S.L. 2000. Impaired cued and contextual memory in NPAS2-deficient mice. Science 288: 22262230.

Gorbacheva, V.Y., Kondratov, R.V., Zhang, R., Cherukuri, S., Gudkov, A.V., Takahashi, J.S., and Antoch, M.P. 2005. Circadian sensitivity to the chemotherapeutic agent cyclophosphamide depends on the functional status of the CLOCK/BMAL1 transactivation complex. Proc. Natl. Acad. Sci. 102: 3407-3412.

Hardeland, R., Coto-Montes, A., and Poeggeler, B. 2003. Circadian rhythms, oxidative stress, and antioxidative defense mechanisms. Chronobiol. Int. 20: 921-962.

Harrison, D.E. and Archer, J.R. 1988. Biomarkers of aging: Tissue markers. Future research needs, strategies, directions and priorities. Exp. Gerontol. 23: 309-325.

Karakelides, H. and Sreekumaran Nair, K. 2005. Sarcopenia of aging and its metabolic impact. Curr. Top. Dev. Biol. 68: 123-148.

Katic, M. and Kahn, C.R. 2005. The role of insulin and IGF-1 signaling in longevity. Cell. Mol. Life Sci. 62: 320-343.

Kennaway, D.J. 2005. The role of circadian rhythmicity in reproduction. Hum. Reprod. Update 11: 91-101.

Kondratov, R.V., Chernov, M.V., Kondratova, A.A., Gorbacheva, V.Y., Gudkov, A.V., and Antoch, M.P. 2003. BMAL1-dependent circadian oscillation of nuclear CLOCK: Posttranslational events induced by dimerization of transcriptional activators of the mammalian clock system. Genes \& Dev. 17: 1921-1932.

Kondratov, R.V., Kondratova, A.A., Lee, C., Gorbacheva, V.Y., Chernov, M.V., and Antoch, M.P. 2006a. Posttranslational regulation of circadian transcriptional CLOCK(NPAS2)/BMAL1 complex by CRYPTOCHROMEs. Cell Cycle 5: 890-895.

Kondratov, R.V., Shamanna, R.K., Kondratova, A.A., Gorbacheva, V.Y., and Antoch, M.P. 2006b. Dual role of the CLOCK/BMALl circadian complex in transcriptional regulation. FASEB T. 20: 530-532.

Kujoth, G.C., Hiona, A., Pugh, T.D., Someya, S., Panzer, K., Wohlgemuth, S.E., Hofer, T., Seo, A.Y., Sullivan, R., Jobling, W.A., et al. 2005. Mitochondrial DNA mutations, oxidative stress, and apoptosis in mammalian aging. Science 309: 481-484.

Kurosu, H., Yamamoto, M., Clark, J.D., Pastor, J.V., Nandi, A., Gurnani, P., McGuinness, O.P., Chikuda, H., Yamaguchi, M., Kawaguchi, H., et al. 2005. Suppression of aging in mice by the hormone Klotho. Science 309: 1829-1833.

Lowrey, P.L. and Takahashi, J.S. 2004. Mammalian circadian biology: Elucidating genome-wide levels of temporal organization. Annu. Rev. Genomics Hum. Genet. 5: 407-441.

McClung, C.A., Sidiropoulou, K., Vitaterna, M., Takahashi, J.S., White, F.J., Cooper, D.C., and Nestler, E.J. 2005. Regulation of dopaminergic transmission and cocaine reward by the Clock gene. Proc. Natl. Acad. Sci. 102: 9377-9381.
Miller, B.H., Olson, S.L., Turek, F.W., Levine, J.E., Horton, T.H., and Takahashi, J.S. 2004. Circadian clock mutation disrupts estrous cyclicity and maintenance of pregnancy. Curr. Biol. 14: 1367-1373.

Monk, T.H. 2005. Aging human circadian rhythms: Conventional wisdom may not always be right. J. Biol. Rhythms 20: 366-374.

Mounkes, L.C., Kozlov, S., Hernandez, L., Sullivan, T., and Stewart, C.L. 2003. A progeroid syndrome in mice is caused by defects in A-type lamins. Nature 423: 298-301.

Nadon, N.L. 2006. Exploiting the rodent model for studies on the pharmacology of lifespan extension. Aging Cell 5: 9-15.

Panda, S., Antoch, M.P., Miller, B.H., Su, A.I., Schook, A.B., Straume, M., Schultz, P.G., Kay, S.A., Takahashi, J.S., and Hogenesch, J.B. 2002a. Coordinated transcription of key pathways in the mouse by the circadian clock. Cell 109: 307-320.

Panda, S., Hogenesch, J.B., and Kay, S.A. 2002b. Circadian rhythms from flies to human. Nature 417: 329-335.

Radak, Z., Chung, H.Y., Naito, H., Takahashi, R., Jung, K.J., Kim, H.J., and Goto, S. 2004. Age-associated increase in oxidative stress and nuclear factor $\mathrm{\kappa B}$ activation are attenuated in rat liver by regular exercise. FASEB J. 18: 749-750.

Razzaque, M.S., Sitara, D., Taguchi, T., St-Arnaud, R., and Lanske, B. 2006. Premature aging-like phenotype in fibroblast growth factor 23 null mice is a vitamin D-mediated process. FASEB J. 25: 25.

Reppert, S.M. and Weaver, D.R. 2002. Coordination of circadian timing in mammals. Nature 418: 935-941.

Rudic, R.D., McNamara, P., Curtis, A.M., Boston, R.C., Panda, S., Hogenesch, J.B., and Fitzgerald, G.A. 2004. BMAL1 and CLOCK, two essential components of the circadian clock, are involved in glucose homeostasis. PLoS Biol. 2: e377.

Shimba, S., Ishii, N., Ohta, Y., Ohno, T., Watabe, Y., Hayashi, M., Wada, T., Aoyagi, T., and Tezuka, M. 2005. Brain and muscle Arnt-like protein-1 (BMAL1), a component of the molecular clock, regulates adipogenesis. Proc. Nat1. Acad. Sci. 102: 12071-12076.

Spanagel, R., Pendyala, G., Abarca, C., Zghoul, T., Sanchis-Segura, C. Magnone, M.C., Lascorz, J., Depner, M., Holzberg, D., Soyka, M., et al. 2005. The clock gene Per2 influences the glutamatergic system and modulates alcohol consumption. Nat. Med. 11: 35-42.

Storch, K.F., Lipan, O., Leykin, I., Viswanathan, N., Davis, F.C., Wong, W.H., and Weitz, C.J. 2002. Extensive and divergent circadian gene expression in liver and heart. Nature 417: 78-83.

Trifunovic, A., Wredenberg, A., Falkenberg, M., Spelbrink, J.N., Rovio, A.T., Bruder, C.E., Bohlooly, Y.M., Gidlof, S., Oldfors, A., Wibom, R., et al. 2004. Premature ageing in mice expressing defective mitochondrial DNA polymerase. Nature 429: 417-423..

Turek, F.W., Joshu, C., Kohsaka, A., Lin, E., Ivanova, G., McDearmon, E., Laposky, A., Losee-Olson, S., Easton, A., Jensen, D.R., et al. 2005 Obesity and metabolic syndrome in circadian Clock mutant mice. Science 308: 1043-1045.

Tyner, S.D., Venkatachalam, S., Choi, J., Jones, S., Ghebranious, N., Igelmann, H., Lu, X., Soron, G., Cooper, B., Brayton, C., et al. 2002. p53 mutant mice that display early ageing-associated phenotypes. Nature 415: 45-53.

Weiss, A., Arbell, I., Steinhagen-Thiessen, E., and Silbermann, M. 1991. Structural changes in aging bone: Osteopenia in the proximal femurs of female mice. Bone 12: 165-172. 


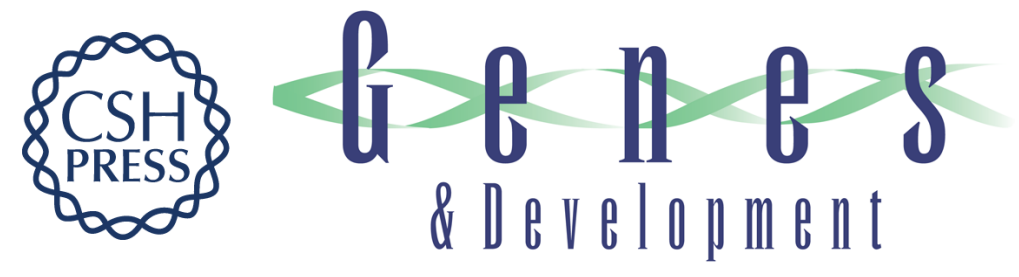

\section{Early aging and age-related pathologies in mice deficient in BMAL1, the core componentof the circadian clock}

Roman V. Kondratov, Anna A. Kondratova, Victoria Y. Gorbacheva, et al.

Genes Dev. 2006, 20:

Access the most recent version at doi:10.1101/gad.1432206

\section{Supplemental http://genesdev.cshlp.org/content/suppl/2006/06/30/20.14.1868.DC1 Material}

Related Content

References

License

Email Alerting

Service
Decreased Life Span in the Absence of the Circadian Clock Component BMAL1

Sci. STKE July, 2006 2006: tw250

This article cites 38 articles, 8 of which can be accessed free at:

http://genesdev.cshlp.org/content/20/14/1868.full.html\#ref-list-1

Articles cited in:

http://genesdev.cshlp.org/content/20/14/1868.full.html\#related-urls

Receive free email alerts when new articles cite this article - sign up in the box at the top right corner of the article or click here.

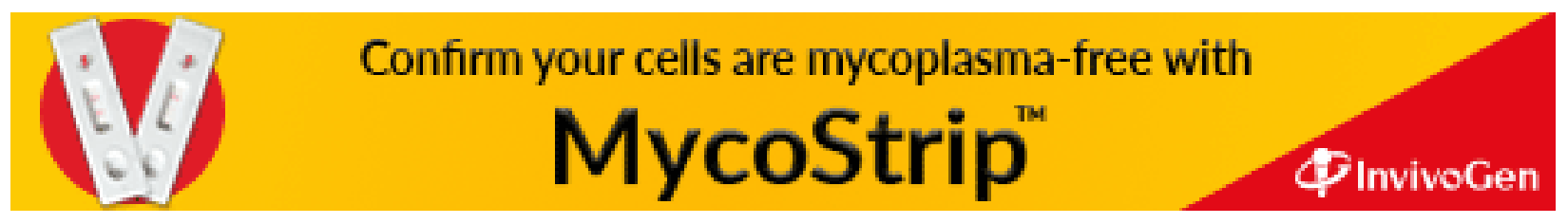

